\title{
Discordancia lipídica y placa carotídea en pacientes obesos en prevención primaria
}

\section{Lipid discordance and carotid plaque in obese patients in primary prevention}

Walter Masson \& $\square$, Daniel Siniawski, Martín Lobo, Graciela Molinero

Consejo de Epidemiología y Prevención Cardiovascular, Sociedad Argentina de Cardiología, Ciudad Autónoma de Buenos Aires, Argentina

Received 03 July 2017, Accepted 07 September 2017

Resumen

Introducción

Los pacientes obesos con discordancia lipídica podrían tener una mayor prevalencia de aterosclerosis subclínica. Los objetivos de nuestro trabajo fueron: 1) determinar la prevalencia de discordancia lipídica en una población de pacientes obesos en prevención primaria; 2) investigar la asociación entre la discordancia lipídica y la presencia de placa aterosclerótica carotídea (PAC).

\section{Métodos}

Se incluyeron sujetos mayores de 18 años obesos (índice de masa corporal $\geq 30 \mathrm{~kg} / \mathrm{m}^{2}$ ) sin enfermedad cardiovascular, diabetes, o tratamiento hipolipemiante, provenientes de 6 centros de cardiología. Se definió "discordancia lipídica» cuando, independientemente del valor de C-LDL, el valor de colesterol no HDL superaba $30 \mathrm{mg} / \mathrm{dL}$ el valor de C-LDL. Se identificó la presencia de PAC por ultrasonido. Se realizaron análisis uni y multivariados explorando la asociación entre la discordancia lipídica y la presencia de PAC.

\section{Resultados}

Se incluyeron 325 pacientes obesos (57,2\% hombres, edad media: 52,3 años). La prevalencia de discordancia lipídica fue del $57,9 \%$. Mostraron PAC el $38,6 \%$ de los pacientes. Esta proporción fue mayor en los sujetos con discordancia lipídica en comparación con los pacientes sin este patrón lipídico ( $44,4 \%$ vs. $30,7 \%, p=0,01)$. En el análisis univariado (OR: 1,80; IC95\%: 1,14-2,87; $p=0,01$ ) y en el multivariado (OR: 2,07; IC95\%: 1,22-3,54; $p=0,007$ ), la presencia de discordancia lipídica se asoció con una mayor probabilidad de presentar PAC.

\section{Conclusión}

En pacientes obesos, la discordancia lipídica se asoció con una mayor prevalencia de PAC. Evaluar pacientes obesos con esta estrategia podría identificar a los sujetos con mayor riesgo cardiovascular residual. 
Palabras clave

Obesidad, Discordancia lipídica, Placa aterosclerótica carotídea

Keywords

Obesity, Lipid discordance, Carotid atherosclerotic plaque 